\title{
EFFECT OF STEP LOADING ON THE LINEAR AND NON-LINEAR VISCOELASTIC BEHAVIOR OF SOLID POLYMERS USING FEM
}

\author{
Abdulamir S. Resen \\ College of Engineering, Nawroz University, Duhok, Kurdistan Region - Iraq
}

\begin{abstract}
Problems of step loading in one and two dimensions were investigated using FEM. 2D FE program was modified to study the linear and non-linear viscoelastic strain using non-linear viscoelastic model "Schapery's model" as constitutive low along with spectrum of material properties. A very carefully selected four case studies covering an important part of viscoelastic behavior were solved for two types of solid polymers, Polyurethane and Poly (vinyl chloride). An excellent fit was obtained when comparing the predicted results with those obtained experimentally.
\end{abstract}

KEYWORDS : Linear Viscoelastic, Non-Linear Viscoelastic.

\section{INTRODUCTION}

The use of polymers as load bearing materials requires an intensive study of their behavior under different loading conditions. In linear viscoelasticity $(\square<0.5 \%)$ Boltzmann superposition theory is always used, but when the strain exceeds this range up to $(\square<2 \%)$ the behavior of polymers becomes nonlinear, so that superposition does not hold any more. It is well known that the linear part of behavior of solid polymers is very limited in practical applications and hence the dominant behavior is the non-linear behavior. One of the most important properties of solid polymers is that they are materials with history, i.e. any contribution of any level of loading at an instant of time will affect the final behavior of solid polymers. So that it is of great importance to study the behavior of solid polymers under complex loading history. Many experimental studies [1,2] were performed for different kinds of polymers under complex loading programs in one and two dimensions. The experimental results were compared with results obtained from the power low for the same cases and a poor fit was obtained ${ }^{[1,2]}$. For this

Academic Journal of Nawroz University

(AJNU) Volume 7, No 3 (2018).

Received 16 April 2018.

Regular research paper : Published 20 June 2018

Corresponding author's e-mail : a.s.resen@yahoo.com

Copyright @2018 A. S. Resen.

This is an open access article distributed under the Creative

Commons Attribution License. reason, a linear finite element program that is used to predict linear and onset of non-linear viscoelastic behavior of polymers [3] is modified in this study using a spectrum of material properties for computing the strain for each time interval. The use of spectrum of properties has improved the accuracy of the results especially in long time creep. In the present study the constitutive low used in the FE program is the nonlinear Schapery's model [4], which is a very accurate model of integral form. So that the finite element formulation used here is linear in order to avoid complexity; but the constitutive law used is non-linear.

\section{Schapery's Model}

Various models have been developed for characterizing the non-linear viscoelastic behavior of solid polymers $[5,6,7]$. It is important to mention here that the model used should be good enough to describe the effects of loading and environment. For this reason, the model used in this study is one of the most important models based on thermodynamic theory in representing linear and non-linear viscoelastic behavior of polymers.

For uniaxial deformation under isothermal condition, the Schapery representation takes the form :

$$
\varepsilon(t)=g_{o} J_{o} \sigma+g_{1} \int_{o}^{t} \Delta J[\psi(t)-\psi(\tau)] \frac{d\left(g_{2} \sigma\right)}{d \tau} d \tau
$$

where $\mathrm{J}_{\mathrm{o}}$ and $\Delta \mathrm{J}(\psi)$ are instantaneous and transient components of creep compliance at linear viscoelasticity respectively, $\psi$ is the reduced time defined by : 


$$
\psi(t)=\int_{o}^{t} \frac{d s}{a_{\sigma}[\sigma(s)]}
$$

and the material properties $\mathrm{g}_{0}, \mathrm{~g}_{1}, \mathrm{~g}_{2}$ and $a_{\sigma}$ are nonlinear functions of stress with $\mathrm{g}_{\mathrm{o}}=\mathrm{g}_{1}=\mathrm{g}_{2}=a_{\sigma}=$ 1.0 when the stress is sufficiently small, or in other words, the material exhibit linear behavior. Effect of temperature and physical aging can be incorporated to the constitutive equation (1) through shift factors that are added to the reduced time [8]. However, such effects are ignored in this study and the reduced time is therefore just equivalent to the real time.

For uniaxial tensile step loading ( $\sigma=$ constant), equation (1) reduced to

$$
\frac{\varepsilon(t)}{\sigma}=J(t)=g_{o} J_{o}+g_{1} \Delta J(t)
$$

By evaluating the linear material parameters $J_{o}$ and $\Delta J_{o}$ and the so-called nonlinearity factors $\mathrm{g}_{\mathrm{o}}$ and $\mathrm{g}_{1}$, a mathematical description that simulate creep behavior is obtained which is valid for wide range of applied stresses and loading times.

\section{Fitting of Material Parameters}

\subsection{Linear Parameters}

The first step is to fit equation (3) to a single experimental curve that lies in the linear range with $g_{o}$ and $\mathrm{g}_{1}=1.0$. For long-term creep experiments, it is sufficient to choose $\mathrm{m}$ retardation times one per decade [9] in the time interval of the experimental curve. For most selected cases in this study $\mathrm{m}$ is chosen $=4$. The linear parameters can be computed by least square technique as follow :

At time $t_{j}$, the calculated compliance is

$$
J_{c}\left(t_{j}\right)=J_{o}+\sum_{i=1}^{m} J_{i}\left(1-e^{-t j / \tau_{i}}\right)
$$

where the transient compliance is expressed in form of Proney series and $\square_{\mathrm{i}}$ is the retardation times. If there are $p$ data points in the experimental curve, denoted by $\mathrm{J}_{e}\left(t_{\mathrm{j}}\right)$, The residual $\mathrm{R}$ is defined as :

$$
R=\sum_{j=1}^{p}\left[J_{c}\left(t_{j}\right)-J_{e}\left(t_{j}\right)\right]^{2}
$$

The least squares problem now is to find the unknowns $J_{o}$ and $J_{i}(i=1 \ldots \mathrm{m})$ which minimize $R$, i.e. $\frac{\partial R}{\partial J_{o}}=0$ and $\frac{\partial R}{\partial J_{i}}=0$ for all $i$

As $J_{e}$ has no dependence on the unknowns, then $\left.\frac{\partial R}{\partial J_{o}}=\sum_{j=1}^{p}\left[J_{c}\left(t_{j}\right)-J_{e}\left(t_{j}\right)\right] \times \frac{\partial J_{c}\left(t_{j}\right)}{\partial J_{o}}=0\right\}$

where, $\frac{\partial J_{c}\left(t_{j}\right)}{\partial J_{o}}=1$

Similarly,

$$
\left.\frac{\partial R}{\partial J_{i}}=\sum_{j=1}^{p}\left[J_{c}\left(t_{j}\right)-J_{e}\left(t_{j}\right)\right] \times \frac{\partial J_{c}\left(t_{j}\right)}{\partial J_{i}}=0\right\}
$$

where, $\frac{\partial J_{c}\left(t_{j}\right)}{\partial J_{i}}=1-e^{-t} j / \tau_{i}$

From equations (7) and (8)

$$
\sum_{j=1}^{p} J_{c}\left(t_{j}\right)=\sum_{j=1}^{p} J_{e}\left(t_{j}\right)
$$

By substituting equation (4) into equation (11), the following expression is obtained

$$
\left.p J_{o}+\sum_{j=1}^{p} \sum_{i=1}^{m} J_{i}\left(1-e^{-t_{j} / \tau_{i}}\right)=\sum_{j=1}^{p} J_{e}\left(t_{j}\right)\right\}
$$

Similarly, from equations (9) and (10)

$$
\left.\sum_{j=1}^{p} J_{c}\left(t_{j}\right)\left(1-e^{-t_{j} / \tau_{i}}\right)=\sum_{j=1}^{p} J_{e}\left(t_{j}\right)\left(1-e^{-t_{j} / \tau_{i}}\right)\right\}
$$

By substituting equation (4) into equation (13), the following expression is obtained

$$
\left.\begin{array}{l}
J_{o} \sum_{j=1}^{p}\left(1-e^{-t_{j} / \tau_{i}}\right)+\sum_{j=1}^{p}\left[\sum_{i=1}^{m} J_{i}\left(1-e^{-t_{j} / \tau_{i}}\right)\right]\left(1-e^{-t_{j} / \tau_{i}}\right) \\
=\sum_{j=1}^{p} J_{e}\left(t_{j}\right)\left(1-e^{-t_{j} / \tau_{i}}\right)
\end{array}\right\}
$$

Equations (12) and (14) represent a linear system of equations that can be expressed as :

$$
[A]\{H\}=[Z]
$$

where $\{\mathrm{H}\}$ is the set of unknowns $J_{o}$ and $J i$ 's.

and $[\mathrm{Z}]$ is a vector calculated from the experimental data points by using the right hand side of equations (12) and (14).

An iterative program that uses Gauss-Seedle method [10] was used for solution.

\subsection{Nonlinearity Factors}

In the nonlinear viscoelastic range, nonlinear factors $g_{0}$ and $g_{1}$ are stress dependent functions that are calculated from at least three distinct uniaxial stress levels. Such factors are determined using, once again, least squares method as follow :

Let the calculated nonlinear compliance at time $t_{j}$ and 
for a certain stress, $\sigma_{k}$, is given by

$$
\left.J_{c}\left(t_{j}, \sigma_{k}\right)=g_{o}\left(\sigma_{k}\right) J_{o}+g_{1}\left(\sigma_{k}\right) \sum_{i=1}^{m} J_{i}\left(1-e^{-t_{j} / \tau_{i}}\right)\right\}
$$

If there are $p$ experimental data points in the nonlinear region, $J_{e}\left(t_{j}, \sigma_{k}\right)$, then the residual is computed as

$$
R=\sum_{j=1}^{p}\left[J_{c}\left(t_{j}, \sigma_{k}\right)-J_{e}\left(t_{j}, \sigma_{k}\right)\right]^{2}
$$

In this case, the unknowns $g_{0}$ and $g_{1}$ are determined by minimizing $R$ such that :

$$
\frac{\partial R}{\partial g_{o}}=0 \text { and } \frac{\partial R}{\partial g_{1}}=0
$$

where the linear parameters are already computed from previous section. After performing the partial derivatives in equation (18) and substituting equation (16) in the resulted equations, the following expressions are obtained

$$
\left.p J_{o} g_{o}\left(\sigma_{k}\right)+\left[\sum_{j=1}^{p} \sum_{i=1}^{m} J_{i}\left(1-e^{-t_{j} / \tau_{i}}\right)\right] g_{1}\left(\sigma_{k}\right)=\sum_{j=1}^{p} J_{n}\left(t_{j}, \sigma_{k}\right)\right\}
$$

and

$$
\left[J_{o} \sum_{j=1}^{p} \sum_{i=1}^{m} J_{i}\left(1-e^{-t_{j} / \tau_{i}}\right)\right] g_{o}\left(\sigma_{k}\right)+\left[\sum_{j=1}^{p}\left\{\sum_{i=1}^{m} J_{i}\left(1-e^{-t_{j} / \tau_{i}}\right)\right\}^{2}\right] g_{1}\left(\sigma_{k}\right)
$$$$
=\sum_{j=1}^{p} J_{n}\left(t_{j}, \sigma_{k}\right) \sum_{i=1}^{m} J_{i}\left(1-e^{-t_{j} / \tau_{i}}\right)
$$

Equations (19) and (20) are simultaneously solved for the values of $g_{o}$ and $g_{1}$ at one particular stress $\square_{\mathrm{k}}$. This procedure is repeated for other stress levels in order to see the variation of nonlinearity factors with respect to the stress. Upon quadratic curve fitting of $\mathrm{g}_{0}$ and $\mathrm{g}_{1}$ versus stress, the following forms of the two functions are obtained

$$
\begin{aligned}
& g_{o}(\sigma)=a_{o}+a_{1} \sigma+a_{2} \sigma^{2} \\
& g_{1}(\sigma)=b_{o}+b_{1} \sigma+b_{2} \sigma^{2}
\end{aligned}
$$

where $a_{0}, a_{1}, a_{2}, b_{0}, b_{1}$, and $b_{2}$ are the constants of curve fitting.

\section{Finite Element Description}

Because the type of the element used in FEM solution is greatly affected by the accuracy of the solution then the choice of element type should be done carefully. In the present study a nine-nodded 2-D isoparametric quadrilateral elements with two degrees of freedom per node as shown in Fig. (1) were used for both polyurethane and PVC. The mesh used for the solution and the boundary conditions for each case is also given for each case study.

\section{Results and Discussion}

The case studies presented in this study are carefully selected to cover a wide range of applications in which polymeric parts may subjected to step loading in one and/or two dimensions. Figure $(2-a, 2-b)$ shows the meshes used in the finite element solution and the boundary conditions for two typical case studies one is uniaxial shear, and the other is combined tensile and shear. The materials used in this study are widely used in engineering applications.

1. One Dimension Two Step Loading :

This case study represents a proper entrance to the subject of complex loading history that any polymeric part may be subjected to. The strain of polyurethane plate resulting due to two step decreasing load history as in Figure (3.a) can be separated into two regions Figure (3.b). The first one represents non-linear creep strain resulting from applying stress of $13.7 \mathrm{MPa}$. The second region, which is the one of interest, represents the interaction of response between the two steps. It represents the recovery resulting from removing the first step load combined with the creep resulting from the second step

2. Two Dimension Two Step Loading:

The loading History of this case study is more complicated and hence two materials Polyurethane foam and PVC were selected with different combination of shear and tensile stresses as shown in Figures (4.a, 4.b) and (6.a, 6.b). It is observed that shear stress gives a shift to tensile creep curve for the two materials as shown in Figure (5.a) this shift appears very clear in case of PVC Figure (7.a) since in the first region there is no tensile stress while tensile strain existed. The tensile stress has a lower effect on shear strain response as could be seen from Figures (5.b) and (7.b). It is clear that the contribution of each component of stress and each step within each component changes the shape of strain curves with a considerable amount and hence affects the final value of the strain at the end of time duration of stress.

3.One Dimension Three Step Loading:

The last case study selected in this paper is PVC plate subjected to three-step loading program as shown in Figure (8.a). This case presents a more complex stress history to investigate this problem intensively. It is clear that the strain resulted can be separated into three regions, Figure (8.b), the first region is pure creep curve, the second region results from the contribution of creep of the second step and recovery of the first step. The third region represents the contribution of creep effect due to second and third steps and recovery due to first step of loading program. 


\section{Conclusions}

The present study concentrates on one of the most important types of loading history in polymer applications, which is the step loading. Two very important features are studied numerically for the first time, which are the effect of each step of load on the behavior of polymeric part when this part is subjected to another step "next loading step". The other important feature is the effect of each step in each component of stress on the behavior of polymeric part when subjected to the next loading step in the same component, and when subjected to another loading component.

The results obtained showed an excellent agreement with the experimental results even for the case of very long time $[1,2]$.

References

1. Findley W.N., J.S.Y. Lai,

"A Modified Superposition Principle Applied to Creep of Non-Linear Viscoelastic Material Under Abrupt changes in State of Combined Stress" Trans. of the Soc. Reheology. Vol.11, No.3, pp. (361-380), 1967.

2. Findley W.N., G. Khosla. "Application of the Superposition Principle and Theories of Mechanical Equation of State, Strain, and Time Hardening of Creep of Plastics Under Changing Loads", J. of Applied Physics Vol.26, No.7, pp. (821-832), 1955.

3. Al-Hadithi Q. T.,

"The Linear and The Onset of Nonlinear Creep Analysis in Solid Polymers using FE Method", M.Sc. Thesis, Mechanical Engineering Department, University of Basrah, 1998.

4. Schapery R.A.,

"On The Characterization of Nonlinear Viscoelastic Materials", Polymer. Eng. and Sc. Vol.9, No.1, 1969.

5. Kitagawa, M. and Takagi, H.,

"Non-Linear Constitutive Equation for Polyethylene under Combined Tension Torsion", J. Polymer Science, part B : Polymer Science, Vol. 28, pp. 1843-1853, 1990.

6. Kichnin, J., Dang Van, K. and Boytard,

"Finite Element Simulation of New Two-Dissipative Mechanisms Model for Bulk Medium Density Polyethylene", J. of Materials Sc., Vol. 31, pp. 1653-1661, 1996.

7. Buckley, C.P. and Johns, D.C.,

"Glass Rubber Constitutive Model for Amorphous Polymers near Glass Transition", Polymer, Vol. 17, No. 17, pp.3301-3312, 1995.

8. Lai, J. and Bakker, A.,

"3-D Schapery Representation for Non-Linear Viscoelasticity and Finite Element Implementation", Computational Mech. Vol. 18, pp.182-191, 1996.

9. Brueller O. S. \& Stiener H.,

“Creep-Based Characterization of Nonlinear Relaxation
Behavior of Plastics", Polymer Engineering \& Science, Vol. 33, No. 21, 1993.

10. Carnham, B., Luther, H. A. and Wilkes J. O.,

"Applied Numerical Methods" John Wiley \& Sons, 1969.

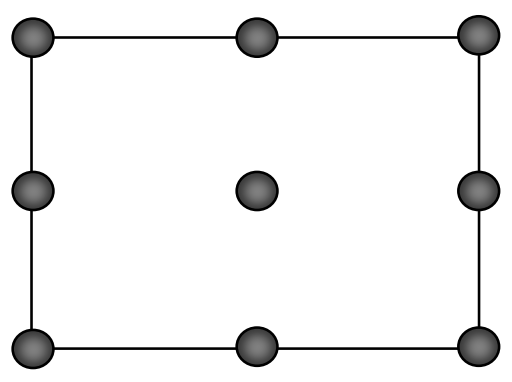

Fig. (1) : 9-Nodded Quadrilateral Isoparametric Element

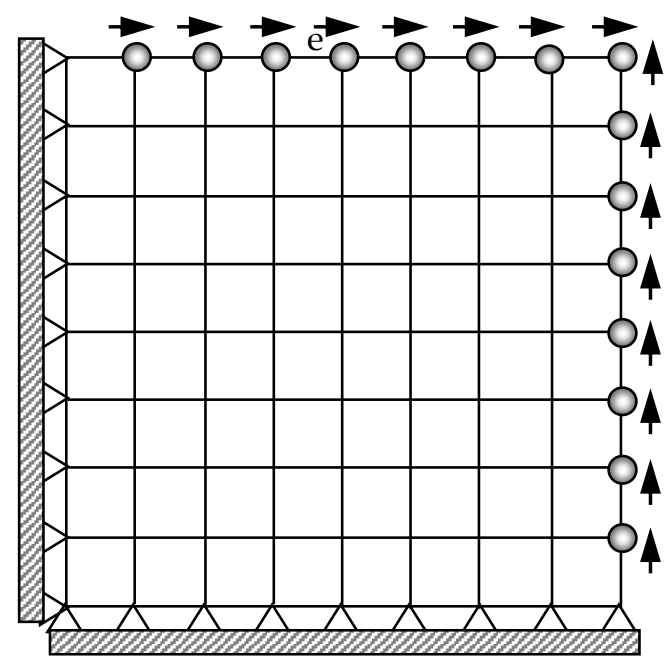

Fig. (2.a) : FEM Mesh with Shear Stress Boundary Conditions

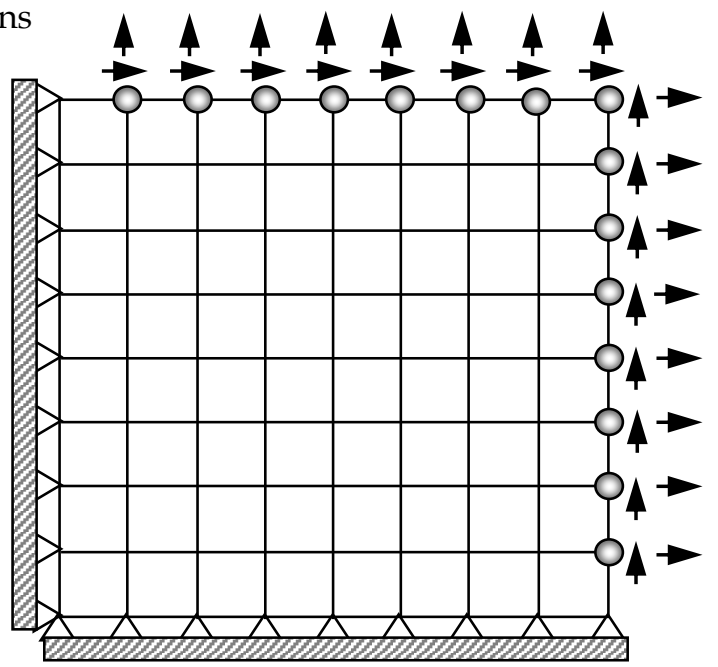

Fig. (2.b) FEM Mesh with Combined Load Boundary Conditions 


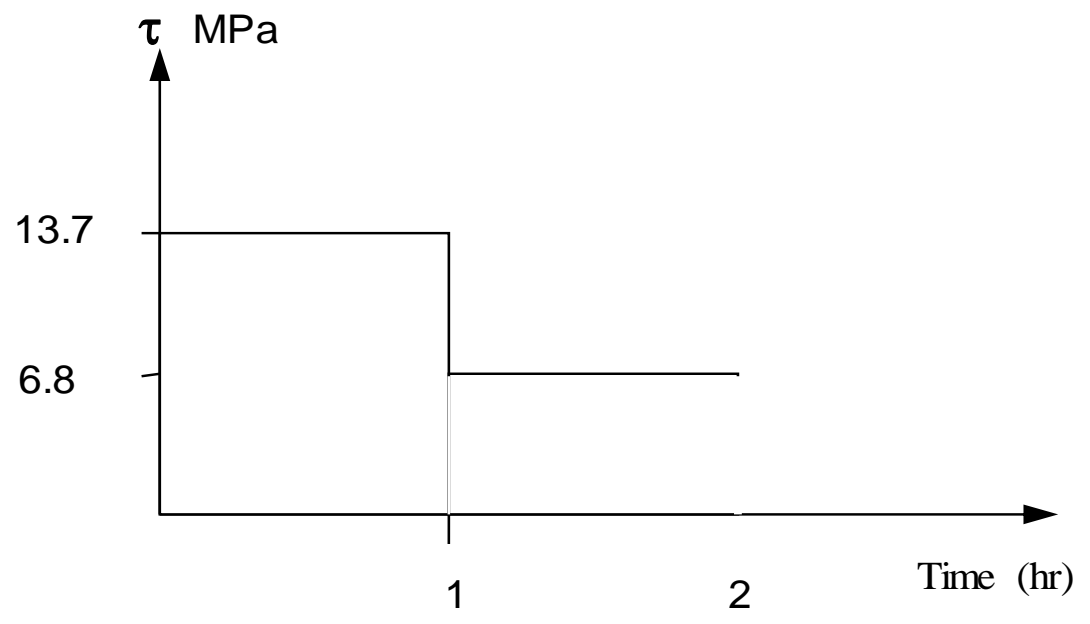

Fig.(3.a) Two Step Shear Loading Program

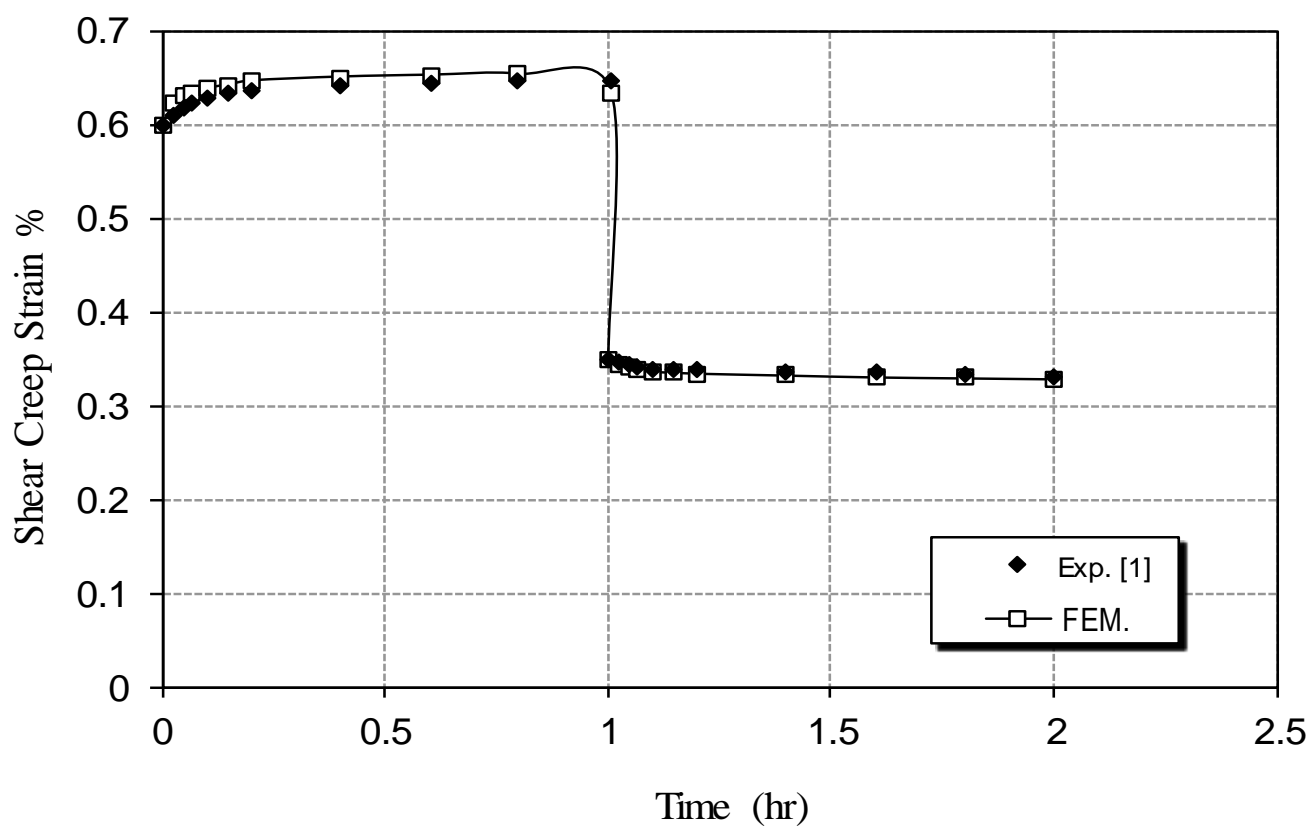

Fig.(3.b) Polyurethene Plate Subjucted to Two Step Shear Stress 


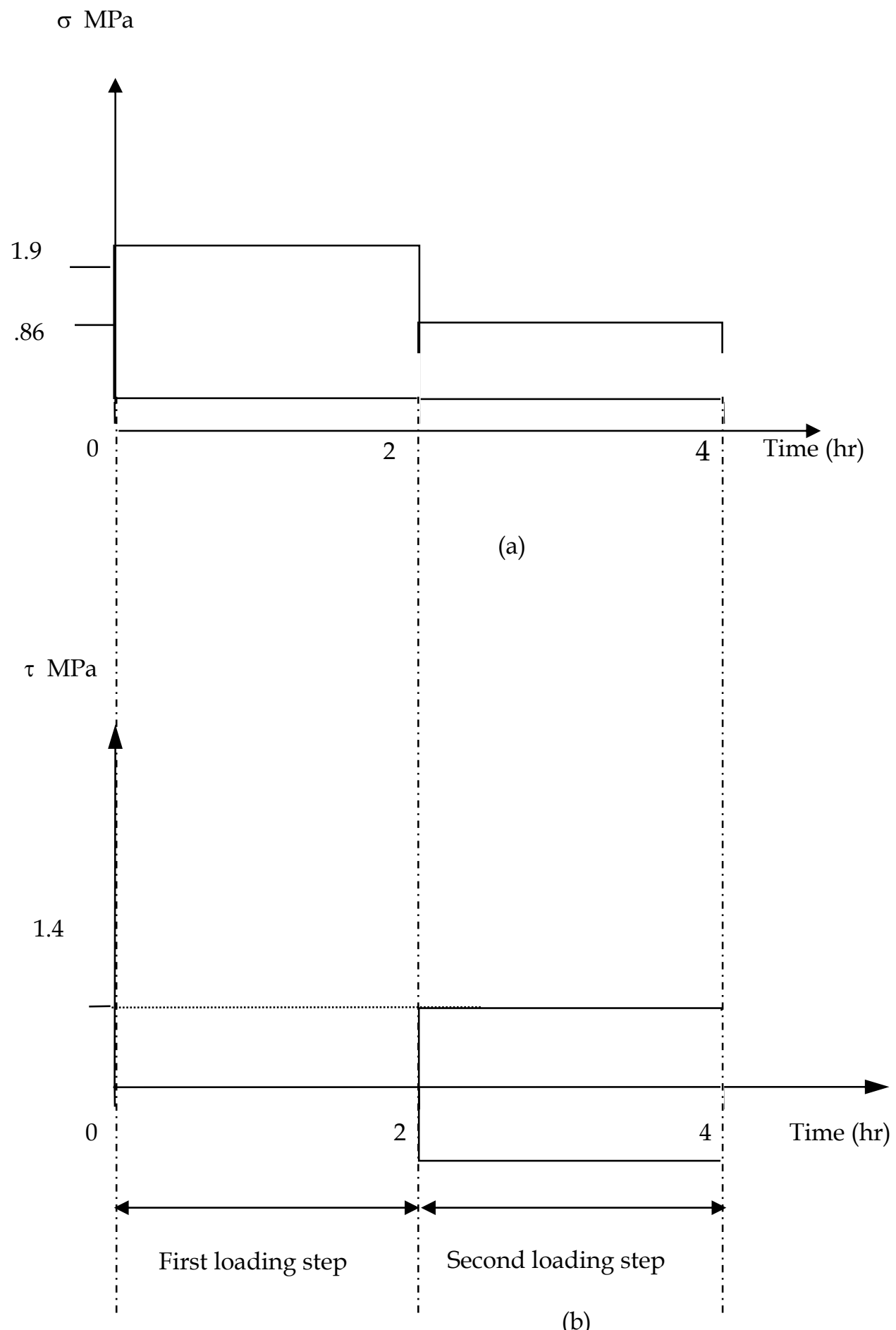

(b)

Fig. (4) Loading Program of Polyurethane plate
a. (Tensile Component)
b. (Shear Component) 


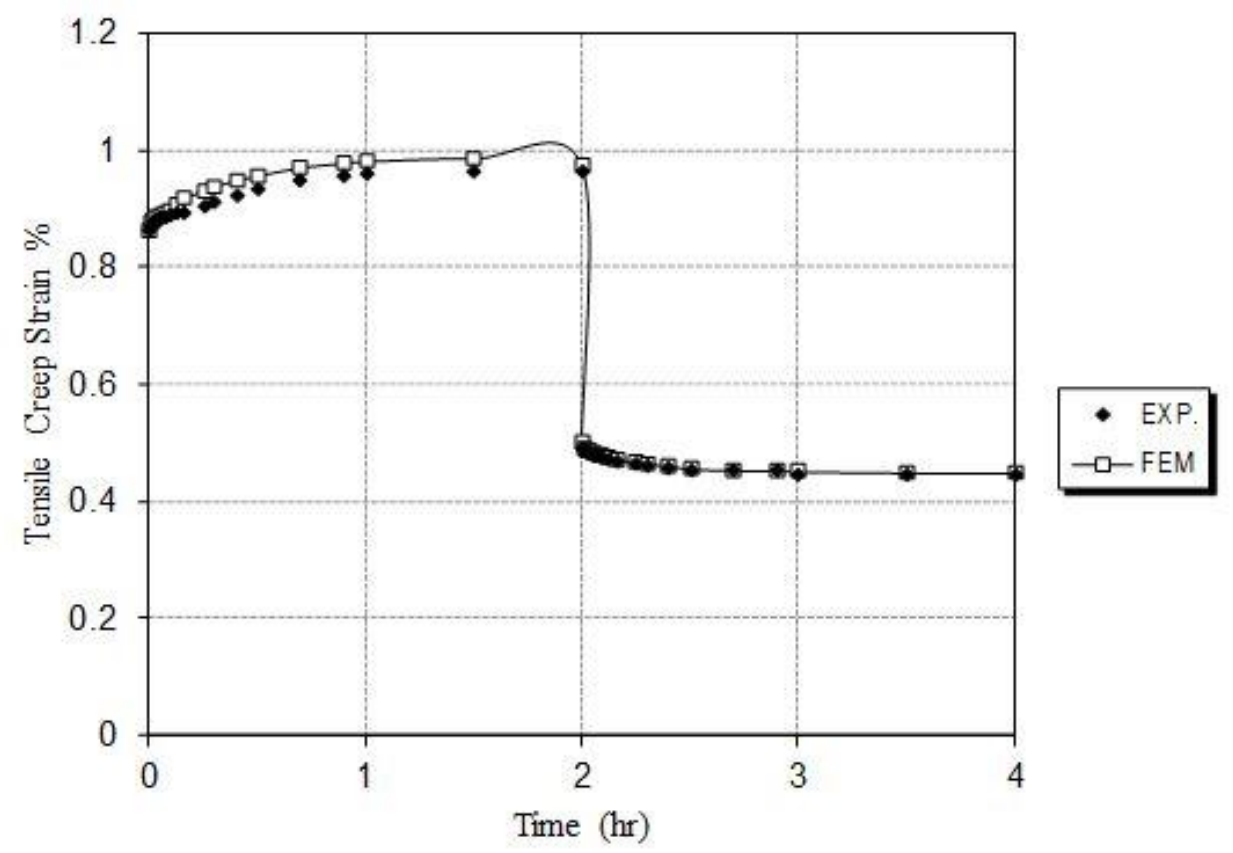

Fig. (5.a) A Rigid Polyurethane Form Plate Subjected to Combined Step Loading Program ( Tensile Component )

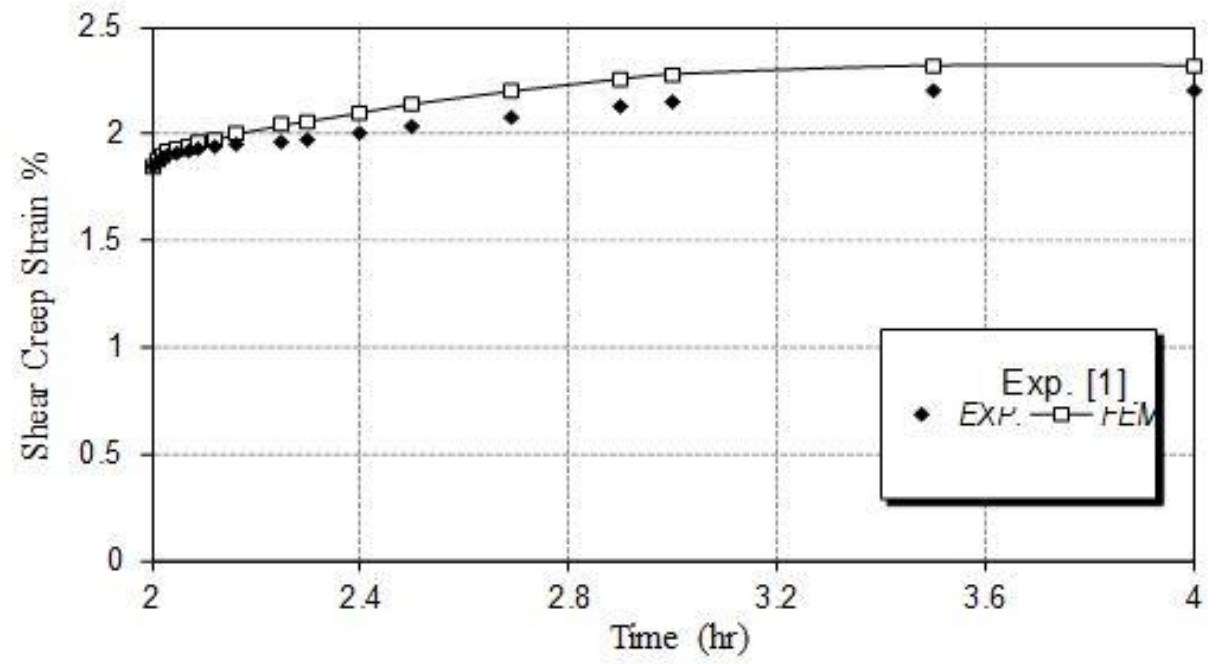

Fig.(5.b) A Rigid Polyurethane Foam Plate Subjucted to Combined step Loading Program (Shear Component) 


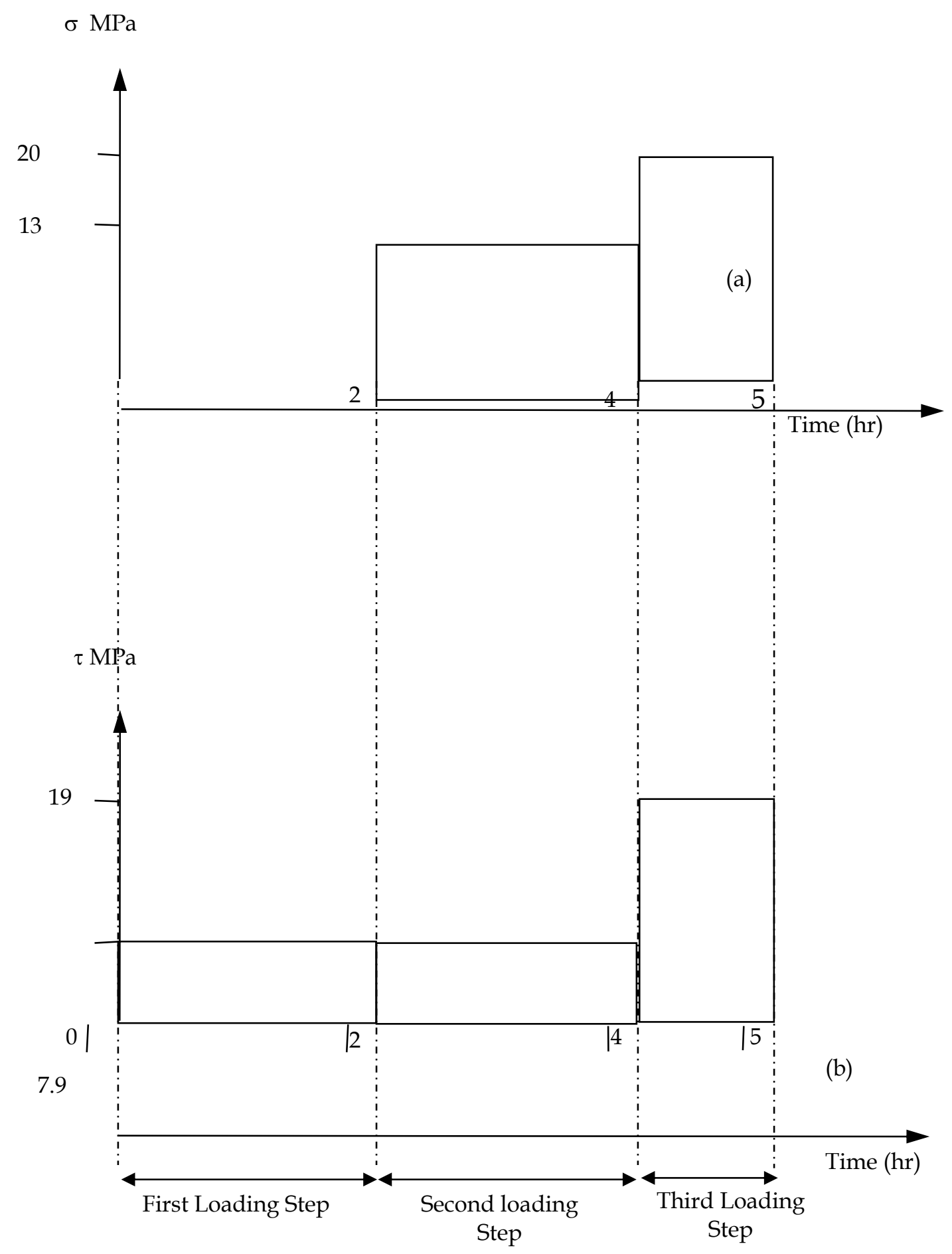

Fig. (6) Loading Program for PVC Plate

a. (Tensile Component)

b. (Shear Component) 


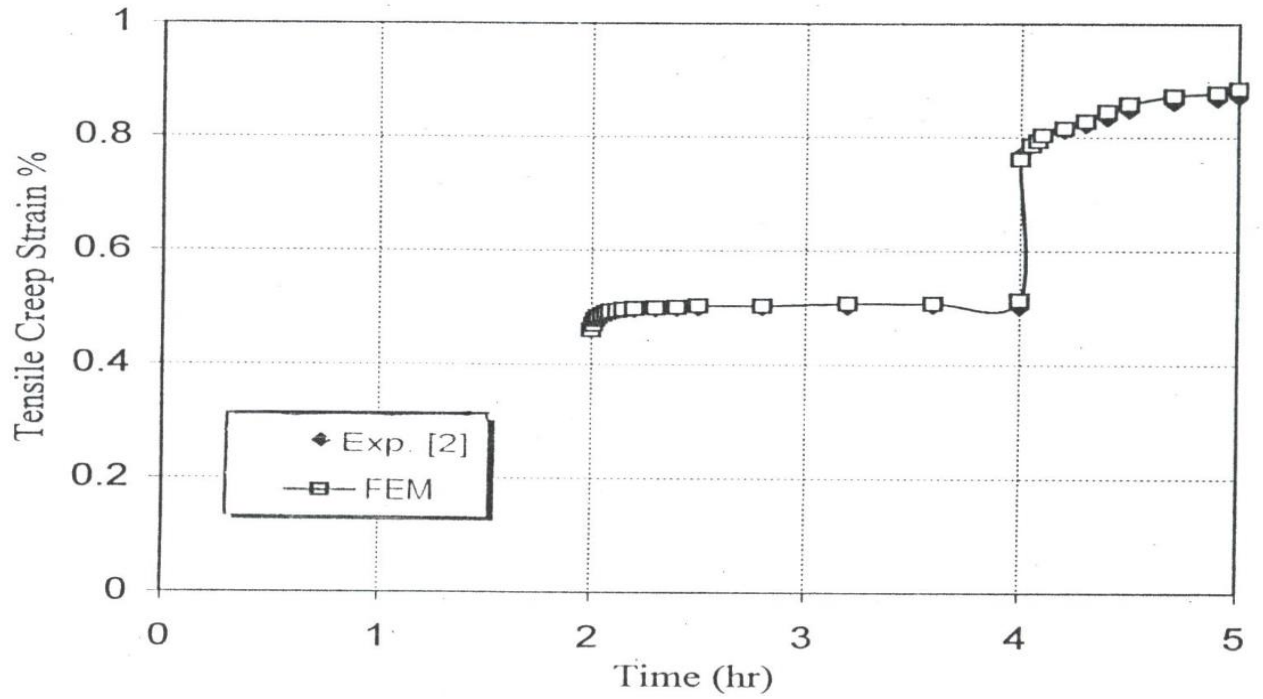

Fig.(7.a)PVC Plate Subjucted to Comined Two Step Load Prgram (Tensile Component)

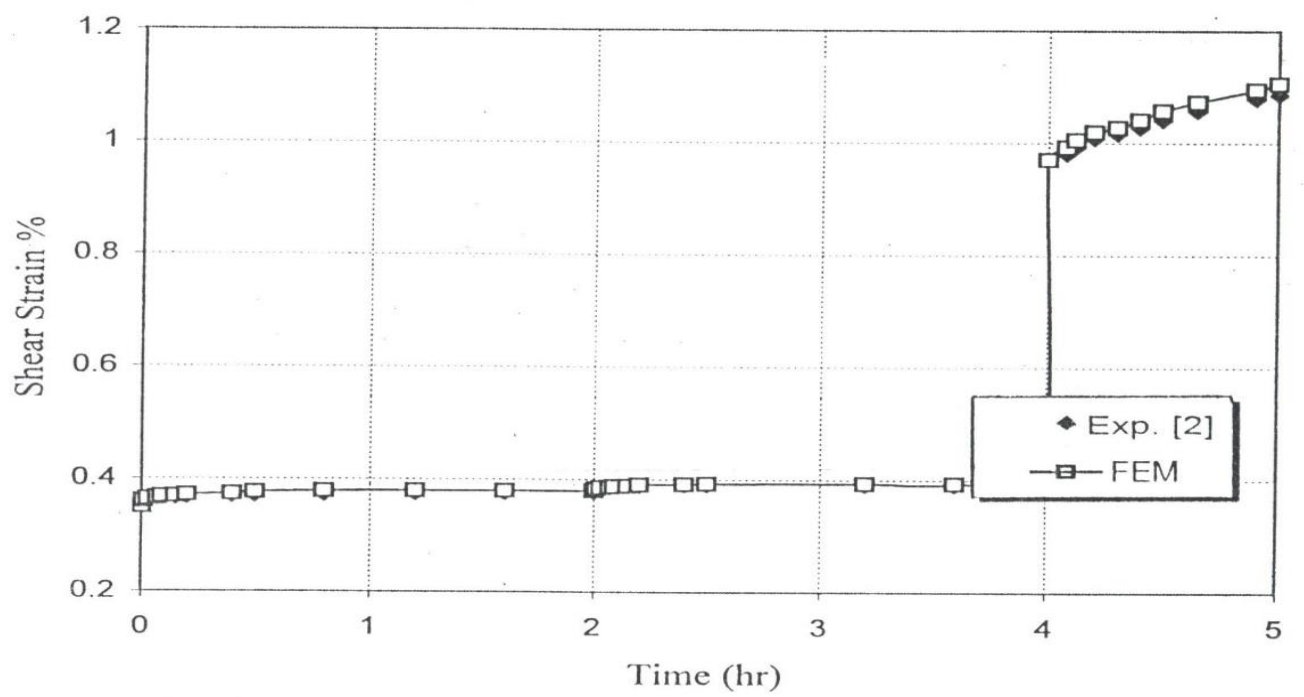

Fig(7.b) PVC Plate Subjucted to Combined Step Loading Program (Shear Component) 


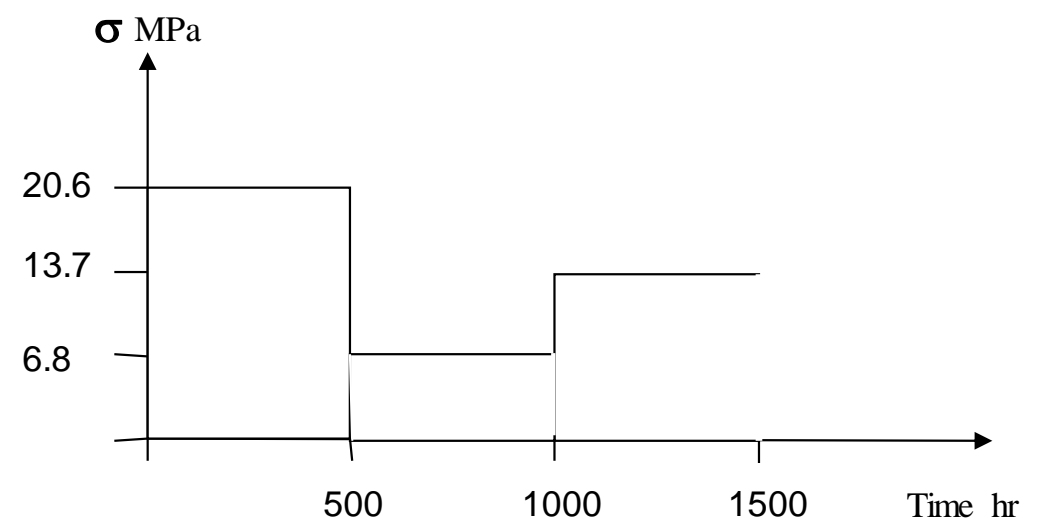

Fig (8.a) 3-Step Loading Program For Long period of Time

(Tensil Loading)

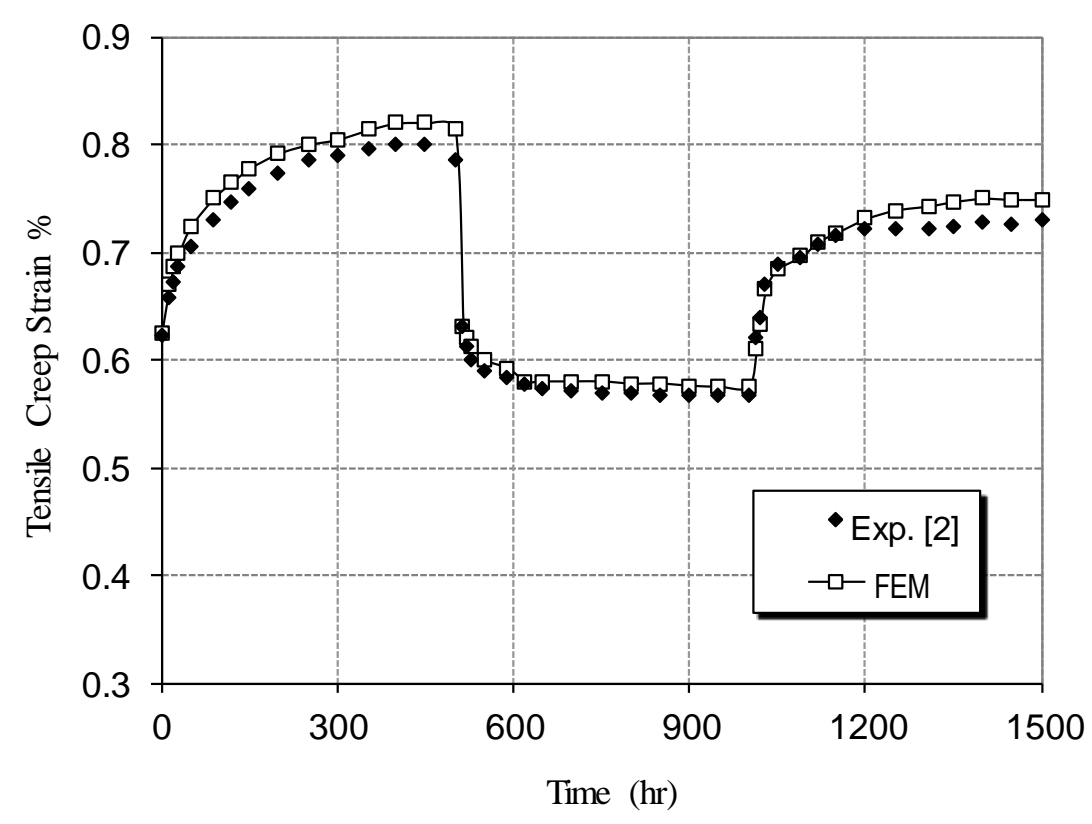

Fig(8.b) PVC Plate Subjucted to Three Step Tensile Load for a Long Time 